\title{
A study of neonatal seizures in view of a etiology onset type and clinical manifestations
}

\author{
Divakar R. ${ }^{1}$, Aravind Thirugnama S. M.,", Subburaman V. Sonraju ${ }^{3}$ \\ ${ }^{1}$ Assistant Professor, ${ }^{2,3}$ Senior Assistant Professor, Dept. of Paediatrics, ${ }^{1}$ Thoothukudi Medical College, Thoothukudi, Tamil \\ Nadu, ${ }^{2,3}$ Thanjavur Medical College, Thanjavur, Tamil Nadu, India
}

*Corresponding Author:

Email: aravindts78@ rediffmail.com

\begin{abstract}
Introduction: Neonatal seizures are significant because most of them are treatable if a specific cause is identified. Prompt diagnosis is important to plan correct treatment. The time of onset of seizures is closely related to the aetiology of seizure

Objectives: The objective of the present study is to know the etiology of seizures, the time of onset, relation to the cause and the common types of seizures Methodology: 102 neonates presenting with seizures admitted in the NICU of RMH, Thanjavur Medical College, Thanjavur during January 2014 to august 2014 were taken for study, Detailed antenatal, natal and post natal history were taken and examination of baby done and HIE staged according to Modified Sarnat's staging. Then relevant investigations were done and etiology of neonatal seizures were diagnosed and staged

Results: In the present study, out of 102 neonates studied, 82 were full-term, among these 60 (58.8\%) were AGA and 22 (21.6\%) were SGA. 19 babies (18.6\%) were preterm. Male: Female ratio in our study was 1.5:1.

Conclusion: The most common etiology for neonatal seizures is HIE. Onset of seizures during first 3 days of life has significant correlation with HIE as etiology. Subtle seizures is the commonest type of seizures and is difficult to identify.
\end{abstract}

Keywords: Neonatal seizures; Birth asphyxia; HIE; Subtle seizures; Hypoglycemia.

\section{Introduction}

Neonatal seizures are common and may be the first manifestation of serious neurological disease. There are various types of neonatal seizures like tonic clonic, myoclonic and subtle seizures. The timing of seizures has relevance since it is closely related to the etiology. A seizure includes clinical phenomena that are temporally associated with EEG seizures activity and therefore are clearly epileptic. The definition also includes paroxysmal clinical phenomenon that often are most associated temporally with EEG seizure activity. Four essential seizure types can be recognized in neonates according to Volpe JJ08 i.e.

(1) Subtle; (2) Clonic; (3) Tonic and (4) Myoclonic ${ }^{5}$. Multifocal refers to clinical activity that involves more than one side, is asynchronous and usually is migratory, whereas generalized refers to clinical activity that is diffuse bilateral, synchronous and non-migratory.

\section{Aims and Objectives}

1. To study the etiology of neonatal seizures.

2. To study the time of onset of neonatal seizures and its relationship with the etiology.

3. To study the various types of presentation of seizures in neonates significant because very few are idiopathic.

\section{Material and Methods}

Methods of Collection of Data: The type of study is analytical study. Sample size was 102 babies with neonatal seizures admitted in SNCU of Thanjavur medical college between January to August 2014. The results were analyzed using chi square test. Ethical approval no.089.

Inclusion Criteria: Neonates (first 28 days of life) presenting with at least one of the following clinical type of seizures:

1. Generalized tonic seizures.

2. Multifocal clonic seizures

3. Focal clonic seizures

4. Myoclonic seizures

5. Subtle activity with apnea or autonomic features. Exclusion Criteria:

6. Seizure like activity (jitteriness, tetanic spasm, etc.) instituted, but also to ensure as meaningful a prognostic statement as possible

7. Subtle activity without apnea or autonomic features detailed antenatal, natal and post natal history were taken as per the proforma enclosed.

\section{Results}

Analysis of Cases and Results: There were 102 neonates admitted to RMH, Thanjavur Medical College, Thanjavur, with convulsions during the period of 8 months from January 2014 to August 2014 Gestational Age: In the present study, out of 102 babies, 82 were full term. Among these 82 full term neonates $60(58.82 \%)$ were appropriate for gestational age and $22(21.6 \%$ were small for gestational age. There were $19(18.6 \%)$ preterm babies and 1 post term baby). In our study, 62 were males and 40 were female babies with male to female ratio of 1.5:1. Out of 102 cases, $13(12.75 \%)$ were born at home and $89(87.25 \%)$ at hospital. Out of hospital deliveries 70 were inborn and 19 were out born. Majority of the neonates in the 
present study were born by spontaneous vaginal delivery in $62(60.8 \%)$ cases. Caesarean section was done in 35 cases $(34.3 \%)$ the indications were prolonged second stage of labor with MSAF in 12 cases $\mathrm{PIH}$ in 4 cases. Forceps delivery were done in 5 cases $(4.9 \%)$ and the indication was prolonged second stage of labour in 4 cases. Antenatal Details: In the present study 8 babies were born to mothers with PROM, 8 babies were born to mothers with PIH, 2 babies were with APH and two and one mother had fever during first and last trimester respectively. Natal Factors: In our study 72 mothers had prolonged 2nd stage of labor (>2 hours), out of which 42 had birth asphyxia. 29 mothers had meconium stained amniotic fluid, out of which 20 had birth asphyxia. In our study onset of seizures on first day of life was seen 37 neonates $(36.27 \%)$, on second day of life 28 neonates developed seizures $(27.45 \%)$, on third day of life $17(16.67 \%)$ babies developed convulsions. The first three days of life together constituted $80.39 \%$ of neonatal seizures. Type of Neonatal Seizures In our study out of 102 neonatal seizures, 96 neonates had one of the 4 classically described neonatal seizures. Among these 46 neonates $(45.1 \%)$ subtle seizures, 36 neonates $(35.4 \%)$ had generalized tonic seizures, $8(7.8 \%)$ had multifocal clonic seizures and $6(5.9 \%)$ had focal clonic seizures. 6 neonates had mixed type of seizures, among these 3 had subtle with generalized tonic seizures and 3 had subtle with clonic seizures. Birth asphyxia is the commonest cause of neonatal seizures in our study. 57 babies had birth asphyxia (55.9\%) and 3 babies had birth asphyxia with hypoglycaemia and 1 baby had hypocalcaemia. 18 babies had hypoglycemia (17.6\%), 13 babies had meningitis $(12.7 \%), 3$ babies had hypocalcemia $(2.9 \%)$. 2 baby had IEM. 3 baby had intraventricular hemorrhage $(2.9 \%) .3$ babies had congenital anomalies as a cause for neonatal seizures $(2.9 \%)$. In 3 neonate, no cause was identified. Chi squared for onset of seizures on first three days and more than three day with etiology $=72.84$ with $\mathrm{p}$ value of $<0.001$ (statistically highly significant for onset of seizures on first three days of life with birth asphyxia). The onset of seizures on first day was seen in 39 neonates, 38 of them were due to birth asphyxia and one was due to congenital anomaly. On 2nd day 22 babies developed seizures and $12(54.54 \%)$ were due to birth asphyxia, 6 were due to hypoglycemia alone, 1 was due to hypocalcemia and 1 was due to meningitis and 2 were due to congenital anomaly. Chi squared for neonates with GTS, Subtle seizures and clonic seizures with etiology $=0.27-$ p $>0.05$ not significant. In present study, out of 57 neonates with birth asphyxia, 28 had subtle seizures, followed by GTS in 19 neonates \& MFC in 4 neonates. In neonates with hypoglycemic seizures, 8 babies had subtle seizures followed by GTS in 6 neonates. In neonates with meningitis (13 neonates), 6 developed subtle seizures and 5 had GTS. In our study there was no correlation between types of neonatal seizures with the etiology $(p>0.05)$ Chi squared for neonates $\geq 2500$ gm \& $<2500$ gm with etiology. $2=16.99$, $\mathrm{P}<0.001$. In the present study, out of 60 term AGA babies, 41 had birth asphyxia, 5 had asphyxia with septicemia, 6 had hypoglycemia, 6 had meningitis, 2 had hypocalcemia, 2 had congenital anomaly and hyperammonemia, hyponatremia with meningitis and unknown cause were seen Out of 22 IUGR term babies, 11 had birth asphyxia, 9 had hypoglycemia, one had hypoglycemia with birth asphyxia and 2 had meningitis. Out of 19 preterm babies, 3 had hypoglycemia, 4 had asphyxia and 1 had hypocalcaemia and 5 had meningitis and 3 had IVH. Out of 1 post-term baby none had birth asphyxia. Low birth weight babies are more prone for seizures due to hypoglycemia with statistically significant $\mathrm{P}$ value of $<0.001$. Birth Asphyxia: Hypoxic ischemic encephalopathy was found to be the commonest cause of neonatal seizures in our study i.e., in 57 cases $(55.8 \%)$. Isolated birth asphyxia was responsible in 53 cases $(51.9 \%)$ and in remaining 3 cases, hypoglycemia was also seen (2.9) and 1 case hypocalcaemia. Type of Delivery: In our study among the 57 neonates with birth asphyxia 30 were born by normal vaginal delivery, among these in the antenatal history $42(73 \%)$ had prolonged second stage of labour, $20(35 \%)$ had MSAF and 8 neonates were delivered at home. 24 neonates were delivered by LSCS, and indication was prolonged second stage of labour with fetal distress in 14 cases $(58 \%)$. 3 neonates were delivered by forceps \& all mothers had prolonged second stage of labour. Day of Onset of Convulsion in Birth Asphyxia: In our study out of 57 cases of birth asphyxia with neonatal seizures, 38 babies developed convulsion on first day of life $(66.6 \%), 12$ babies $(21 \%)$ developed seizures on second day of life, 6 babies $(10.5 \%)$ developed seizures on 3rd day and one baby developed seizures on 4th day. Majority of babies with birth asphyxia developed seizures within first 72 hours of life and more so within first 24 hours of life. In the present study, out of 57 neonates with birth asphyxia, 28 neonates $(49 \%)$ had subtle seizures, 19 babies $(33.3 \%)$ had GTS, 4 babies $(7 \%)$ had multifocal clonic seizures, and 4 babies had focal clonic seizure. Subtle 28 49\% GTS $1933.3 \%$ Multifocal clonic 4 7\% Focal clonic $47 \%$ Mixed type $23.5 \%$ Perinatal and Antenatal factors: In our study in neonatal seizures with birth asphyxia (57cases) 42 mothers had prolonged second stage of labour, 20 mothers $(33.3 \%)$ had meconium stained liquor, 6 mothers had PROM and 5 mothers had PIH.

Metabolic Disorders: Hypoglycemia: Hypoglycemia was the second most common cause of convulsions in neonates in our study. There were 18 babies with hypoglycemia (excluding complicated hypoglycemia associated with birth asphyxia) who presented with seizures. 6 babies with hypoglycemia had onset of seizures on 2 nd day, 5 had onset on third day, 3 on 
fourth day and 1 each on 5th, 6th, 2 on 7 to 28 days. Majority of hypoglycemic convulsions occurred on 2nd day $(33.3 \%)$ followed by 3rd $(27.75 \%)$ Out of 18 babies, 9 were small for gestational age babies $(50 \%), 6$ were term AGA babies and 3 were preterm babies. Hypocalcemia: In present study there were 3 babies with hypocalcemia who presented with seizures. One baby presented on second day with seizures i.e., early onset hypocalcemia. One presented in third day and the other during 4th week (late onset hypocalcemia). Others: In our study one baby had hyponatremia with neonatal meningitis, presented during 2nd week of life. Two babies had hyperammonemia, presented during 2nd week. Neonatal Meningitis: 13 babies were diagnosed as neonatal meningitis in our study and was the third most common cause of neonatal seizures. One case had hyponatremia with neonatal meningitis. Two neonates presented with seizures on 3rd day, 4 neonate presented between 4th to 7 th days and remaining 6 neonate presented between 2nd to 4th week of life. In babies with meningitis 6 babies had generalized tonic seizures, 3 had subtle seizure, one had multifocal clonic seizures and 3 had mixed seizures. Out of 13 babies with neonatal meningitis, 3 were born at home and 1 mother of a baby had fever before and during delivery. Out of 13 babies with Neonatal meningitis, 2 were IUGR babies. Intracranial hemorrhage: In the present study only 3 preterm babies of 32 weeks of gestation developed convulsions, diagnosed by ultrasound of cranium. Congenital anomalies: A female baby presented on 1 day with subtle seizures had hydrocephalus presenting with macrocephaly and protruded eyes. A male baby presented with convulsions (multifocal clonic type) on 2 nd day. The baby had prominent frontal and parietal eminence with short unequal limbs, simian crease and microcephaly. A case with meningomyelocele presented with subtle seizure on 6th day of life. The diagnosis was not known in a patient presenting with seizures (GTS) All the investigations were within normal limits. Mortality: Mortality in our study was $17.6 \%$ (18 cases) and birth asphyxia was the commonest cause seen in 10 cases $(55.5 \%)$.

\section{Discussion}

In the present study 102 neonates with seizures were studied in 6 months period. Both intramurally and extramurally delivered babies were included in the study. Birth asphyxia constituted the primary cause in our study which had a predominant term population. Similar observations was seen in study by Ravneet Sandhu et al ${ }^{7}$ where term babies outnumbered preterm by 81 to $19 \%$. In our study $21.6 \%$ were SGA babies which was similar to a study by sahiba Rima et al which had an SGA population of 20\% Sex Distribution: The sex distribution was 1.5:1 in our study whereas in a study by Lakra Mahaveer et al it was 2:1 Type of Delivery: In our study majority of neonates with seizures were born by normal vaginal delivery $(60.8 \%)$ followed by LSCS (34.3\%) and outlet forceps delivery $(4.9 \%)$. In a study of neonatal seizures by Lakhra Mahaveer et al $68.7 \%$ were born by normal vaginal delivery, $28.1 \%$ by LSCS and $3.1 \%$ by forceps delivery. Perinatal Factors: In the present study, out of 57 cases of birth asphyxia 42 had prolonged and difficult second stage, 20 had MSAF and 5 had PIH. Myles TD etal ${ }^{10}$ found slightly higher incidence of birth asphyxia with prolonged second stage of labour compared to normal second stage of labour. Day of Onset of Seizures: In our study 82 out of 102 neonates with seizures had onset within first three days of life, among these $63 \%$ had onset of seizures within first 2 days life and $36.3 \%$ had onset of seizures within first day of life. 20 neonates $(19.5 \%)$ had onset of seizures after 3 days of life, In a study of neonatal seizures by Ronen Gabriel et al onset of seizures on first day of life was $36 \%, 64 \%$ had onset of seizures within first 48 hours, and $83 \%$ within first week of life, which is similar to our study. Type of Neonatal Seizures: In the present study 46 (45\%) babies had subtle seizures either in the form of lip smacking, cycling movements or apnea with hypertension. Generalized tonic seizures was observed in $35.4 \%$ i.e., 36 cases followed by multifocal clonic in 8 cases $(15 \%)$, focal clonic seizures in 6 babies $(5.88 \%)$ and 6 babies had mixed type of seizures. In a study of neonatal seizures by Brunquell Philip $\mathrm{J}$ et al subtle seizures were the commonest occurring in $51 \%$ (27 of $53)$, followed by focal clonic (42\%), multifocal clonic $(30 \%)$ and GTS (23\%). Lakra Mahaveer et al ${ }^{14}$ also reported that subtle seizures were the commonest. But in a study of neonatal seizures by Soni Arun et al generalized tonic seizure was commonest type of seizure, followed by subtle seizures. Etiology of neonatal seizures: Birth asphyxia is the most common cause of neonatal seizures in our study (57 of 102 cases - 55.8\%) followed by metabolic seizures in which hypoglycemia was the commonest in 18 babies (17.6\%), 3 babies had hypocalcemia. Neonatal meningitis was seen in 13 babies (12.7\%). Congenital anomaly and hyperammonemia were seen in 3 babies each. Birth asphyxia is the commonest cause of neonatal seizures in studies by Soni Arun et al seen in $76.9 \%$ of cases and Ronen Gabriel et al seen in $40 \%$ of cases. According to most of the studies birth asphyxia is the commonest cause of neonatal seizures followed by metabolic or infectious causes. Intracranial hemorrhage constitute small percentage of seizures. Day of onset of seizures and etiology: In our study neonatal seizures during the first two days were mostly due to birth asphyxia and onset of seizures due to birth asphyxia during first three days of was seen in 56 cases $(98.2 \%)$ with highly significant $p$ value of $<.0 .001$. Seizures due to hypoglycemia begin during second day and peaks on third day and again decreases as the days go on. In our study, out of 18 cases of hypoglycemia6 (33\%) began convulsing on 2 nd day of life. At the end of first week, 
seizures are mostly due to neonatal meningitis, which also extends to early second week and later. After first week, onset of seizures are less likely and are mostly due to meningitis and late onset hypocalcemia in our study. In a study of neonatal seizures by Rose Arthur L et $\mathrm{al}^{15}$, majority of babies with perinatal anoxia convulsed on first day of life $(5 / 10-50 \%)$, hypoglycemic neonates convulsed on second and third day $(5 / 7-71 \%)$, majority of neonates with CNS infection convulsed at the end of first week and early second week $(9 / 13-69 \%)$ and babies with hypocalcemia present with convulsions during first and second day of life $(6 / 28)$ and again during late first week and second week (19/28). Birth asphyxia usually presents with seizures within first three days of life, preferably within first 48 hours. Hypoglycemia presents on second and third day, as there is depletion of glycogen stores. Hypocalcemia presents on first and second day if it is early onset hypocalcemia and later i.e., late first week and second week, if it is late onset hypocalcemia. Neonatal meningitis presents with seizures during late first week and second week. Type of neonatal seizures \& Etiology: In the present study $49 \%$ of neonatal seizures with birth asphyxia had subtle type of seizures, followed by GTS in $33.3 \%$ \& MFC in $7 \%$. In neonates with hypoglycemic seizures, 8 babies had subtle seizures \& 6 had GTS. In neonates with meningitis 6 developed subtle seizures and 5 had GTS. There was no correlation between type of neonatal seizures with etiology in our study with $p>0.05$. In study of neonatal seizures compared with EEG studies by Mizrahi EM et al8 GTS \& subtle seizures were likely to be caused by diffuse pathologic processes such as HIE. Clonic seizures, were more likely to be associated with focal or regional lesions such as infarction or ICH with $\mathrm{p}=0.0047$. Birth asphyxia: Birth asphyxia is staged according to Sarnat as HIE-I, II and III and convulsions are common in HIE-II neonates ${ }^{12}$. In our study $99 \%$ of birth asphyxia had HIE-II. In our study 24 babies $(42.1 \%)$ with birth asphyxia had subtle seizures followed by generalized tonic seizures seen in 16 neonates $(28 \%)$, focal convulsions are less likely because Kellaway ${ }^{16}$ Peter8, out of 38 asphyxiated babies, $12(31.6 \%)$ had subtle seizures, followed by tonic seizures in 7 (18\%). Metabolic Disorders: Hypoglycemia: 18 babies had hypoglycemic seizures in our study. 9 were SGA babies, 3 were preterm babies and 6 were term babies i.e., hypoglycemia is more common in low birth weight babies and correlation is statistically significant with $\mathrm{p}<0.001$ as there is depletion of glycogen storage and inadequate feeding during early post-natal days. The day of onset of hypoglycemic seizures were most commonly seen on second, third and fourth day of life in our study (14 of $18 ; 77.7 \%$ ). The diagnosis of hypoglycemia should not be delayed, as it can lead to brain damage. ${ }^{17}$ In a study of hypoglycemia by Lilien Lawrence D et a $41 \%$ of hypoglycemic neonates were SGA babies
Hypocalcemia: In our study 3 babies had hypocalcemia, 2 babies had onset of seizures in 3 days i.e., early onset hypocalcemia, which is due to depleted calcium stores in the baby either due to prematurity and IUGR or complicated hypocalcemia in which other factors like birth asphyxia and maternal diabetes precipitates the hypocalcemia. 1 had late onset hypocalcemia i.e., presented after 3 days of life. This is due to feeding of neonates with phosphate rich milk e.g., cow's milk, formula feeding. Hypocalcemia should be diagnosed early and treated with IV calcium. In a study by Cockburn $\mathrm{F}$ et al serum calcium was low in neonates who were top fed, than the babies who were breastfed. Other metabolic disorders: Hyponatremia and hypernatremia: One case of hyponatremia associated with neonatal meningitis had seizures in our study. Hyponatremia may result from SIADH following CNS infection and birth asphyxia. In our study, 2 case of hyperammonemia was diagnosed and was urea cyclic disorder. Other inborn errors of metabolism also present with seizures in the neonatal period usually in the first week of life and should be suspected when all other etiologies are ruled out or if there is a family history. Intracranial hemorrhage: In our study, 3 preterm baby presented with seizures diagnosed as IVH on ultrasound cranium and CSF study. Preterm neonates are prone for intraventricular hemorrhage because of fragile blood vessels and ineffective supporting structure for periventricular blood vessels. CNS infection: Neonatal meningitis is one of the important causes of neonatal seizures. In our study 13 babies had meningitis $(12.7 \%) .7$ were early onset, which presented on or before 7th day remaining were late onset, which presented after 7th day of life. Most common organisms grown in CSF culture in our study was Staphylococcus aureus followed by E. coli, one baby had hydrocephalus shown by ultrasound of cranium. Tushar Parikh B et al, showed that late onset meningitis is more common than early onset meningitis. Congenital and developmental disorders: These are rare causes of neonatal seizures. In our study, 3 patients had seizures due to congenital anomalies, meningomyelocele, hydrocephalus and the other multiple anomalies with microcephaly Mortality: Mortality in our study was $17.6 \%$ (18 cases) and birth asphyxia was the commonest cause seen in 10 cases $(55.5 \%)$. Mortality in studies by Sandhu Ravneet et al 59 and Ronen Gabriel et al63 were $11.25 \%$ and $9 \%$ respectively and birth asphyxia was the commonest cause.

\section{Conclusion}

Neonatal Seizures typically signal underlying significant neurological disease and represent nonspecific response of the immature nervous system to varied insults. Neonatal seizures are unique \& distinctive when compared to seizures in adults because of the immaturity of the nervous system and require separate classification. The recognition of the etiology 
for the neonatal seizures is often helpful with respect to prognosis and treatment. The most common etiology for neonatal seizures is hypoxic-ischemic encephalopathy. HIE is frequently associated with perinatal complications like prolonged second stage of labour, MSAF and unsafe home deliveries. Most of these are preventable if proper antenatal \& perinatal care is given to the mother. Neonatal seizures themselves in addition to etiology for the seizures, have a significant impact on the developing brain. Therefore, it is critical to recognize neonatal seizures early, at least clinically where continuous video EEG monitoring facilities are not available, and to initiate the treatment. Hypoglycemia \& Hypocalcemia which are one of the commonest causes, should be suspected \& detected as early as possible \& treatment started, before it can cause any additional brain damage. Hypoglycemic seizures is more common in LBW babies. Treatable causes should be evaluated before standard anticonvulsants. The time of onset of neonatal seizures, is significantly associated with the etiology (eg: onset of seizures within first three days is significantly associated with birth asphyxia). Subtle seizures are commonest type of clinical seizure, which is difficult to identify, therefore careful observation of at risk newborns is necessary for the diagnosis. Most of the neonatal seizure are associated with perinatal complications continued advances in perinatal and neonatal medicine may reduce seizure incidence in the future and prevent the neurologic, cognitive and epileptic consequences of neonatal seizures

Funding: No funding sources.

Conflict of interest: None declared.

\section{References}

1. Holmes Gregory L, Khazipov Roustem, Beni-Ari Yehezkiel. New Concepts in neonatal seizures. Neuro Report 2002;31(1):A3-A8.

2. Rosenstein Von. Diseases of children and their remedies. British Edition, 1776.

3. Brett EM. Epilepsy \& Convlusions, In: Textbook of Pediatric Neurology, Churchill Livingstone, New York, 1983:275-276.

4. Victor Maurice, Ropper AH. Epilepsy and Other Seizure Disorders, In: Adam \& Victors' Principles of Neurology, $7^{\text {th }}$ Edition, McGraw Hill, USA (New York), 2001;331332.

5. Evans David, Levene Malcom. Neonatal seizures. Arch Dis Childhood 1998;78(1):70F-75F.

6. Singh Meharban. Neurological Disorders In: Care of the Newborn, $5^{\text {th }}$ Edition, Sagar Publications; New Delhi 2002:340.
7. Rennie Jannet, Boylan Geraldine. Neonatal seizures and their treatment. Curr Opinion Neurol 2003;16(2):177-81.

8. Volpe JJ. Neonatal seizures. Current Concepts and Revised Classification. Pediatr 1989;84(3):422-28.

9. Mizrahi M, Kellaway Peter. Characterization and classification of neonatal seizures. Neurol 1987;37:183744.

10. Watanabe Kazuyoshi et al. Apneic seizures in the newborn. Am J Dis Child 1982;136:980-84.

11. Volpe JJ. Neonatal Seizures. Textbook of Neurology of Newborn, $3^{\text {rd }}$ Edition; WB Saunders Philadelphia; 2002:181-182.

12. Brunquell Philip $\mathbf{J}$ et al. Prediction of outcome based on clinical seizures type in newborn infants. $J$ Pediatr 2002;140(6):707-12.

13. Lakra Mahaveer, Vilhekar KY, Chaturvedi Pushpa. Clinico-biochemical profile of neonatal seizures in a rural medical college. In: Fernandez A, Dadhich JP, Saluja S, Editors, Abstracts, XXIII Annual Convention of National Neonatology Forum. 2003:18-21; Hyderabad. 2003:12122.

14. Bernes Saunders M, Kalpan Allen M. Evolution of neonatal seizures. Pediatr Clin N Am 1994;41(5):107273.

15. Finer $\mathrm{NN}$ et al. Hypoxic ischemic encephalopathy in term neonates: Perinatal factors and outcome. J Pediatr 1981;98(1):112-17.

16. Fenichal Gerald M. Hypoxic ischemic encephalopathy in newborn. Arch Neurol 1983;40:261-66.

17. Volpe JJ. Cerebral blood flow in newborn infant: Relation to hypoxic-ischemic brain injury and periventricular hemorrhage. J Pediatr 1979;94(1):170-73.

18. Lou Hans C, Larsen Niels A, Hansen Bent Friis. Impaired autoregulation of cerebral blood flow in the distressed newborn infant. J Pediatr 1979;94(1):118-21.

19. Sarnat HB, Sarnat MS. Neonatal encephalopathy following fetal distress: A clinical and EEG study. Arch Neurol 1976;33:696-705.

20. Haase Roland et al. Acute subdural hematoma after caesarean section. A case report. Pediatr Critical Care Med 2003;4(2):246-48.

21. Soul Janet S. Intracranial hemorrhage. In: Manual of Neonatal Care. Lippincott Wlliams \& Wilkins Philadelphia, $5^{\text {th }}$ Edition; 2004;523-27.

22. Tsiantos Alex et al. Intracranial hemorrhage in the prematurely born infants. J Pediatr 1974;85(6):854-59.

23. Papile LA et al. Incidence and evolution of subependymal and intraventricular hemorrhage: A study of infants with birth weight less than $1500 \mathrm{gm}$. J Pediatr 1978;92:529.

How to cite the article: R. Divakar, S. M. Aravind, Sonraju S. A study of neonatal seizures in view of a etiology onset type and clinical manifestations. IP Int J Med Paediatr Oncol 2018;4(3):93-7. 